\title{
A Systematic Approach to Improving the Performance of Spiral Antenna
}

\author{
Rahmad Hidayat ${ }^{1}$, Rushendra ${ }^{2}$, Ellisa Agustina ${ }^{2}$, Ike Yuni Wulandari ${ }^{3}$, and Robbi Rahim ${ }^{4}$ \\ ${ }^{1}$ Department of Electrical Engineering, STT Mandala, Bandung, Indonesia \\ ${ }^{2}$ Faculty of Computer Science and Faculty of Engineering, Universitas Mercubuana, Jakarta, Indonesia \\ ${ }^{3}$ Department of Electrical Engineering, Universitas Nurtanio, Bandung, Indonesia \\ ${ }^{4}$ Department of Computer Engineering, Institut Teknologi Medan, Indonesia \\ \{rhidayat4000,rushendra.rustam,ellisa.agustina,ikeyunipp\}@gmail.com; usurobbi85@zoho.com
}

Keywords: Frequency Independent, Optimization, Performance, Spiral Antenna

Abstract: The frequency independent antenna has become a major driver of intensive research and development of spiral antennas in generating large bandwidth. In this paper, a new approach will be adopted that will identify and analyze the wideband spiral antenna design with its various properties and look for opportunities for the application of various forms of novel advanced spiral antennas with improved performance. The results of identification and analysis increasingly show that the improvement of spiral antenna performance is highly dependent on design optimization; and continuous assessment of how techniques, methods, and experiments are possible for the performance improvement of existing spiral antenna properties.

\section{INTRODUCTION}

In the 1950 s a spiral antenna was introduced as a frequency independent antenna, in its sense that its input impedance and gain stay almost regular throughout the bandwidth. Now, this antenna is widely applied in areas such as military, satellite, telemetry, the global positioning system (GPS), wearable, medical, wireless and other wireless communications. Spiral antennas have an area bandwidth of about 10: 1, providing circular polarization with low-profile geometry. The required broadband characteristics can be satisfied by spiral antennas, especially with respect to input impedance and radiation patterns. The antenna radiation pattern is a mathematical function in a graphical form that describes the antenna radiation properties as a spatial coordinate function. (Balanis, 2005) There have been numerous studies on spiral antenna radiation characteristics with different geometric shapes such as circular, rectangular and certain eccentric forms. (Kunisz, 2013) A relatively easy impedance matching, better radiation efficiency, high spectral efficiency, consistency gain, high bandwidth and the main benefit of free frequency are the advantages of this spiral antenna so that it is widely applied in many fields. (Patil et al., 2016)

There are still many properties that are likely to continue to be developed in the process of improving spiral antenna performance, either increased bandwidth, increased gain, voltage standing wave ratio (VSWR), circular polarization improvements, etc. The problem is how to explore the possibilities of new techniques, methods, and experiments in adapting different forms of spiral antennas with improved performance.

\section{ANTENNA GEOMETRY AND OPERATION}

There are several types of spiral antennas including the type of equiangular introduced by J. D. Dyson in 1959 and the sinus antenna found by R. H. Duhamel in 1982, as well as the Archimedean spiral antenna. (Kunisz, 2013)

\subsection{Theoretical Background}


The spiral antennas are a type of ultra wide band (UWB) antenna, circularly polarized, and can be made with machines in a microstrip environment and are a very suitable choice because of their frequency independent nature. There is an antenna class whose pattern and impedance are practically independent of frequency for all frequencies above the minimum cut-off value.(Balanis, 2005) The general formula for this frequency independent antenna form is:

$$
r=e^{\alpha\left(\varphi+\varphi_{0}\right)} F(\theta)
$$

with $\mathrm{r}, \theta$, and $\varphi$ are spherical coordinates, $\alpha$ and $\varphi_{0}$ are constants, and $F(\theta)$ is a theta function. For such circumstances, the frequency change is equivalent to the antenna spin around $\theta=0$. The importance of frequency independent of the spiral antenna is that the frequency change only rotates the active region, the radiating area, along with the spiral arm. As long as the length of arm is sufficient, any frequency can spread effectively. Therefore, scaling factor, $\alpha$, determines the spiral arm's length and consequently the lower cut-off frequency of the antenna, allowing the frequency independent antenna to be scaled according to the desired frequency response. By choosing a scaling factor large enough to achieve the desired electrical performance, it enables making designs with better existing design performance at lower cost. (Hutchinson, 2015)

Spiral Archimedean is one of the spiral classes including parabolic spiral and hyperbolic spiral. The general equation for the Archimedean spiral in spherical coordinates has the equation:

$$
r=a \varphi^{1 / n}
$$

With $\mathrm{n}=1$, an Archimedean spiral is generated, where arm width and spacing are constant as the length of the arm increases. With $\mathrm{n}=2$, a Fermat spiral (parabolic spiral) is produced where the arm spacing decreases as a function of radius or length of the arm. (Hutchinson, 2015)

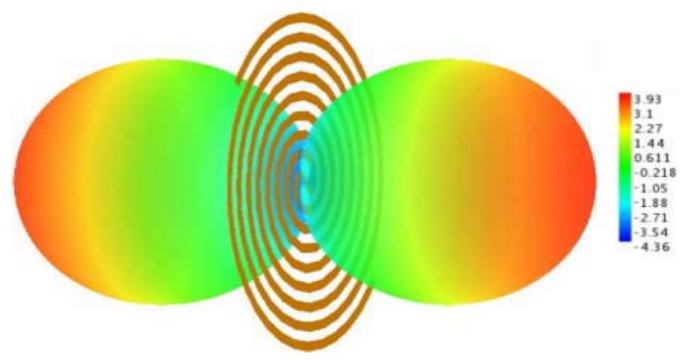

Figure 1: Bidirectional radiation pattern of the Archimedean spiral. (Hinostroza, 2013)

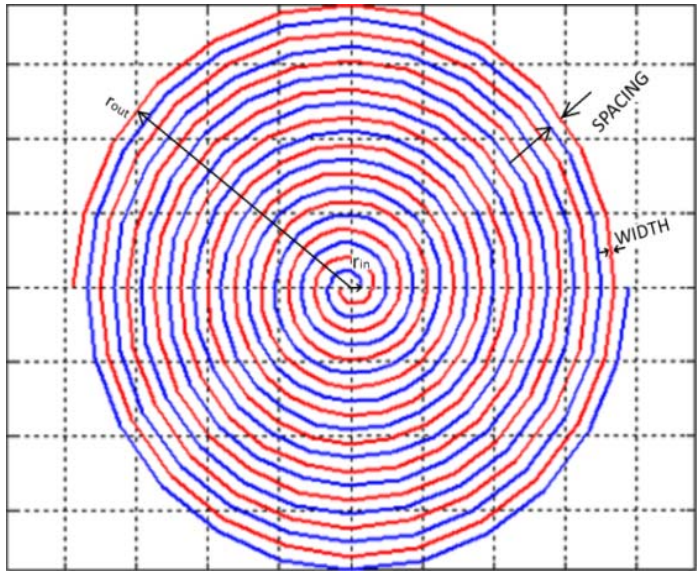

Figure 2: The Archimedean spiral with 10 turns; 2 arms (self-complementary).

The Archimedean spiral antenna forms its own complementary antenna, where the width of the arm and the spacing distance are the same. In theory, this self-complementary antenna has an input impedance of $188 \Omega$. However, the experimental data show that the impedance ranges from $120 \Omega$ to $200 \Omega$. It is generally known that Archimedean spiral antennas produce large main beams and are perpendicular to the spiral plane. The broad broad bidirectional beam can be converted to beam unidirectional by giving a backing span of the ground plane. (Kunisz, 2013) With its ability to maintain consistent gain and input impedance and excellent performance in circular polarization, Archimedean is the most popular type of spiral antenna. In it there are three types of waves are the waves running, fast wave and leaky waves. A wavelength is formed on a spiral arm, enabling broadband performance; fast waves occur due to clutch phenomena between the spiral arms; while leaking waves occur due to energy leakage during propagation through the spiral arms to produce radiation. (Patil et al., 2016)

Hinostroza et al. (2011) summarize that with wide bands, the Archimedean spiral becomes a good candidate in the wideband planar array design. With various shapes (Archimedean, square, star, hexagonal, sinuous, equiangular, etc.), the spiral can be loaded with resistive, capacitive or inductive loads as well as a support cavity to improve performance cross-polarization rejection (XpolR) and voltage standing wave ratio, and the grating lobe limits the highest frequency of the antenna, both of which are related to the dimensions and spacing of the spiral antenna. 


\subsection{Antenna Design}

Theoretically, the design of antenna array will increase the total gain and the improvement of the spiral radiation pattern. An Array of multi-turn spiral antennas can be made of 2, 4 arms, and so on. Design analysis becomes one of the most important factors in realizing spiral antennas with the best performance, either in the initial design manually or with the help of certain simulation tools. For example, when a configuration requires the use of a vertical balun. Problems arise due to their long dimensions and are not suitable for low profile spiral antenna geometry. To solve this problem, Patil et al. (2016) propose a spiral antenna with a physical design method of parallel field structure to expand the bandwidth. This can keep the VSWR low so that the performance of a better antenna for ultra-wide band (UWB) on a very large bandwidth. Gain and VSWR spiral antenna are strongly influenced by antenna dimension, substrate thickness, dissipation factor and the air gap between a ground plane and antenna. The wavelength at the lowest operating frequency will determine the diameter of the spiral antenna, consequently, the size of the antenna is too large to meet the demand of practical size at a low working frequency. In (Liu et al., 2008) investigated a compact Archimedean spiral antenna with a gap loading technique to lower the initial resonance frequency so that it is expected to minimize the spiral dimension.

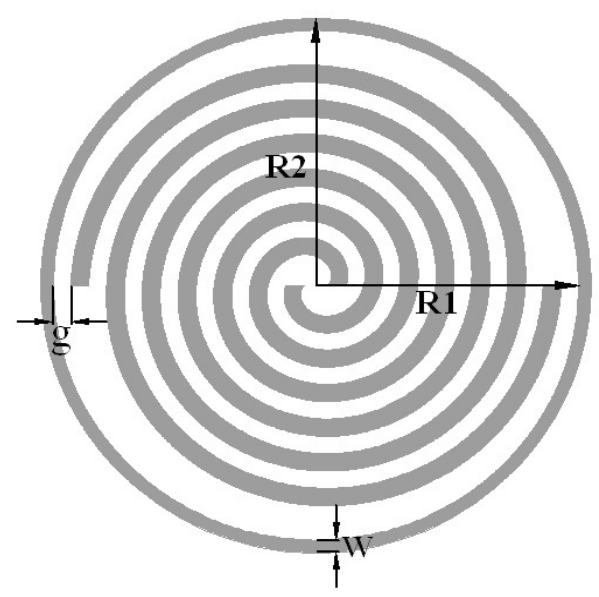

Figure 3: Gap loading for a spiral antenna. (Liu et al.,2008)

In general, applications with spiral antennas require unidirectional coverage, so the effort to eliminate the backlobe energy. Methods performed by several studies in the form of placement of a cavity below the spiral (backed). (Stutzman and Thiele, 1998) Backed cavity reflects cross-pol wave in the backward direction, consequently forward gain increases. Classically the cavity is accomplished by the addition of an electromagnetic absorber which then for the narrowband requirement is enhanced by the addition of a flat metal plate as deep as $\lambda / 4$ below the spiral. Another method exists that puts the metal ring structure of not the same size behind the active area. The buffer cavity can be made of a frequency selective surface (FSS) as did Mohamad et al. (2015) where it reflects signals on the $7-10 \mathrm{GHz}$ band with losses $<0.25 \mathrm{~dB}$ and emits signals on the $3-6 \mathrm{GHz}$ band. The two layers of FSS on this metal plate can also compress the backlobe radiation pattern as well to increase the Archimedean spiral antenna gain. These results simultaneously improve the existing impedance matching. Multilayer dielectric absorbers can also be used in the cavity to obtain unidirectional beam. (Rahman et al., 2011) For the other Archimedean broadband spirals, hybrid-cavity backed electromagnetic band-gap (EBG) structures and a perfect electronic conductor (PEC) are obtained, resulting in 10: 1 lossless bandwidth. (Liu et al., 2010) The loaded-EBG substrate (LEBG) can serve as any planar reflector of any type of antenna with a ground plane to improve the radiation characteristics and the incipient input impedance. (Schreider et al., 2007) There is also an EBG and an artificial magnetic conductor (AMC) structure for certain aircraft applications (Baby and Michael, 2015). Then to increase half power beam width (HPBW) and axial ratio, Archimedean spiral antennas can be designed with various forms of cavities (Klimya \& Prakash, 2015)

In terms of excitation of spiral antennas, the unbalanced architecture with square spiral geometry forms can be one of the design alternatives. Like the two-arm spiral antenna contained in (Morais \& Silva, 2013), where one arm is driven by the inner conductor and the other is connected short-circuited with the ground plane through the cavity. The proposed antenna is capable of generating a wide frequency range for UWB applications on VSWR 2: 1 . The unbalance mode is a simple two-armed Archimedean spiral catheter system supported by a conductive cavity. It is analyzed in (Nakano et al., 2009) where only one arm works, while the other arm is parasitic and without a balance-unbalance (balun) circuit.

The reflective current from the antenna end of the antenna is also another issue of spiral antennas. In order to reverse the polarization of reflected 
energy according to the original polarization of the emitted wave, there is a dual-polarized spiral antenna configuration design in the form of two sets of spiral arms with a set printed on the upper side and another spiral set printed on the underside of the planar PCB. This results in a higher total antenna gain and better polarization purity. (Kunysz, W.) Then the addition of resistive load on the spiral arm can be another solution to the reflection current problem so as to improve the axial ratio of the antenna. (Alhayali, 2012)

The monopole spiral antenna design with highspeed connectors operating at 2.93 to $7.25 \mathrm{GHz}$, the $\lambda \boldsymbol{g} / 4$ monopole width provides an impedance and without balun impedance. The antenna is designed when tested, working in 8 bands of the frequency with a total bandwidth of $1.4 \mathrm{GHz}$, making it suitable for cognitive radio applications and beam switching in certain directions, eg for increased gain toward access point. (Dongre and Takle, 2014) Then a quadratic spiral design printed on a semi-conical surface above the ground perfect electronic conductor (PEC) is performed in (Munoz et al., 2013) for the application of a radiotelescope VLBI2010 with pure circular polarization in the frequency band 2 to $14 \mathrm{GHz}$.

\subsubsection{Computer Aid Simulation}

Modeling and simulation with certain computer tools in most studies results in proximity to existing experimental results. Calculations with the transmission line model include the widely used. Meanwhile, the widely used computer aids are certain commercial 3-D electromagnetic simulators such as IE3D, Sonnet, Ansoft High-Frequency Structure Simulator (HFSS), Antenna Magus, EZNEC, FEKO, Computer Simulation Technique Microwave (CST MW) Studio and others. Each simulator has its own features, for example, CST MW with GUI interface features and various numerical techniques such as time domain, domain frequency, integral equations, asymptotic mode and Eigenmode. Matlab for certain cases is also widely used.

For example, CST MW Studio is used to design and simulate a ring-shaped absorber stacked in the cavity to reduce the reflection of waves from the antenna backing wall cavity. (Kim et al., 2013) The model design and simulation of a tapered multistep matching impedance to a wideband spiral antenna was performed in (Baby and Michael, 2015) using CST MW to obtain VSWR and good gain. CST
MW base on finite integration technology (FIT) is used in (Liu et al., 2008).

Numerical calculations can be presented for example in (Rupčić et al., 2016) to investigate the effect of three different Archimedean spiral distributions on radiation patterns of spherical antenna arrangements with one, two and three distribution arms. The number of elementary antennas all of the conformal arrangements is different from each other. Then here the method of moment (MoM) is used as the method of analysis where the matrix elements are calculated in the spectrum domain as the development of the MoM. This type of antenna array offers a variety of various possibilities of radiation patterns through the distribution of antennas and phase controls. Meanwhile, FEKO software based on MoM is used in (Alhayali, 2012) to study and test radiation pattern and antenna reflection factor (S11) in the range 5,5-6,8 GHz. In (Rahman et al., 2011) FEKO is also used to design Archimedean spiral 2-18 GHz antennas with multilayer dielectric absorbers behind unidirectional generating cavities.

In (Du et al., 2009) simulation of electromagnetic field distribution and the specific absorption rate (SAR) of microstrip spiral antenna in phantom muscle tissue by the finite element method (FEM) was performed. Wire width, the spacing between circles and number of circles compared to each other to see the effect of spiral antenna performance.

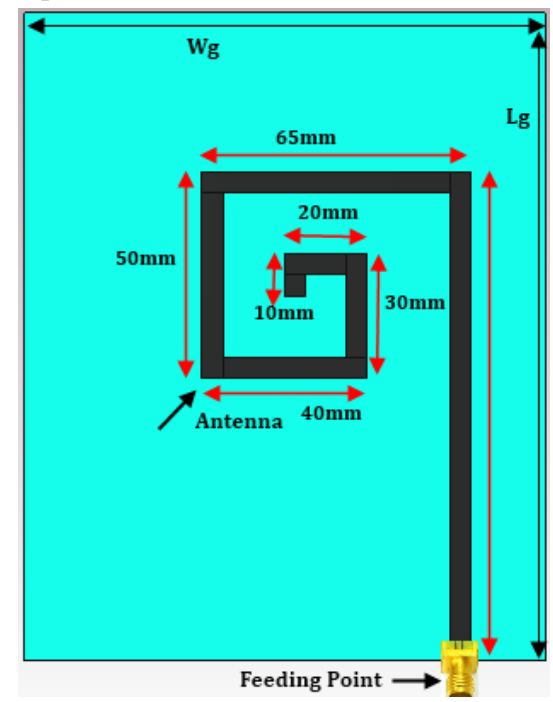

Figure 4: The monopole spiral antenna. (Dongre \& Takle, 2014) 


\subsubsection{Optimization}

The optimization method was introduced to find the shape and size of the antenna in a more structured way so that the antenna design is obtained faster. If done conventionally, this variation in size and/or shape and electromagnetic calculation is a heavy task in the design because if a given shape and size of the antenna cannot meet the given target, it should be tried with other shapes and sizes until the target is reached. In the optimization method introduced cost function, which in the process of calculating the electromagnetic value obtained. Often the cost function is defined as how close the value obtained to the target, then the optimization will be minimized this function during the calculation process. (Alaydrus, 2015) In (Shamii et al., 2012) mentioned there are two types of optimization techniques that help effectively search for the optimum value of antenna parameters. This is shown in Table 1. Another example of soft computing technique for optimization like backpropagation of artificial neural network is found in (Hidayat, 2016).

Table 1: Optimization techniques in antenna design. (Shamii et al., 2012)

\begin{tabular}{|c|c|}
\hline Local & Global \\
\hline $\begin{array}{l}\text { Quasi-Newton Method, } \\
\text { Conjugate Gradient } \\
\text { Methods, Gauss-Newton } \\
\text { Method, Simplex, and } \\
\text { Interior-point Methods }\end{array}$ & $\begin{array}{l}\text { Darwinian } \\
\text { (evolutionary } \\
\text { programming, evolutionary } \\
\text { strategies, genetic } \\
\text { algorithms, differential } \\
\text { evolution), Particle Swarm } \\
\text { Optimization, Simulated } \\
\text { Annealing, Ant Colony } \\
\text { Optimization, Invasive } \\
\text { Weed Optimization }\end{array}$ \\
\hline
\end{tabular}

In the design optimization process, Patil et al. (2016) mention that the spiral antenna design can be optimized by varying the number of turns, the distance between the rotation and the width of the arm. In other words, the spiral antenna is a diminished antenna due to its roll. Kim1 et al. (2012) state that with turn number optimization the gain, return loss and the axial ratio can be improved. While feed position optimization and turn count can improve narrowband multi-resonance.

In (Rupčić et al., 2016) we have studied the various layout of loxodromic antennas based on the influence of Archimedean spiral configuration on the radiation pattern of antenna ball arrangement. The result found that parameter a has a considerable influence on the radiation pattern, where $a$ is a real number of spiral curve equation and it is assumed that $\mathrm{a}>0$. By lowering the value from $\mathrm{a}=0.25$ to $\mathrm{a}=0.15$ then the side lobe level is much better and can differentiate in $4 \mathrm{~dB}$ from the worst case. The optimization results show that by replacing some parameters of spiral constants for each type of distribution and antenna density, the radiation pattern can be improved.

In other configurations optimization by increasing the axial ratio and reducing arm length can help in improving antenna performance. (Patil et al., 2016) In the arrangement of Archimedean spiral antennas, multi-turn four arms change the distance between the arms and the number of turns. (Baheti et al., 2012) The application of genetic algorithm optimization is exemplified in (Rahman et al., 2011) to obtain the best geometry of the absorbent cavity. The optimized design for the wideband application improves the main performance properties, maximizes co-polarization gain, and minimizes the antenna cross-polarization gain across the operational bandwidth.

\subsection{Material and Fabrication}

Different types of substrate materials are selected as needed. Some of which are widely used such as FR4 substrate with dielectric constant $\left(\varepsilon_{\mathrm{r}}\right)$ 4.3-4.9 with thickness of $1.54 \mathrm{~mm}$ and loss tangent $\delta=0.025$; Rogers / Duriod RT 5870 or RT 5880 with $\varepsilon_{\mathrm{r}}$ of 2.2; 2.33 ; 4.33 and a thickness of 1.57-1.8 mm; The $\varepsilon_{\mathrm{r}}$ price selection, in this case, affects the spiral dimension, where the theoretical low price can produce small dimensional sizes. While fabrication is generally done by an etching process.

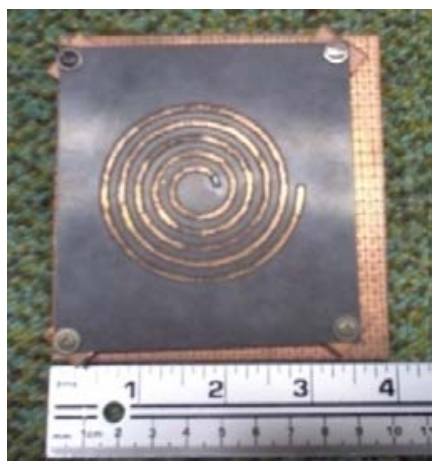

Figure 5: Prototype. (Alhorr, 2007)

\subsection{Measurement}

Measurements of commonly fabricated antennas are made using a widely used measuring instrument Vector Network Analyzer of various brands. 


\section{RESULTS AND DISCUSSION}

In terms of design methods, dimensions and materials become an important issue to get better antenna performance. Whether it's the dimensions of the patch sizes; spiral patch distance to a ground; as well as other supporting dimensions such as backing cavity, parallel field drawing, monopole spiral antenna architectural design, impedance matching construction and balanced-unbalanced if necessary. For example, the physical distance between the spiral arms can be maintained theoretically as far as $0.25 \lambda$ against the ground plane to produce a good performance on the return loss aspect, VSWR, axial ratio, radiation pattern, and of course the gain. In the use of EBG as an example, the dimensions of the width and thickness of the EBG substrate affect the performance of spiral antennas. A more advanced alternative is used photonic band gap (PBG). Then the spacing change between the arms and the turning turns has a lot of influence on the input impedance, gain and multiband properties of the planar spiral arrangements in the range of $1-2.5 \mathrm{GHz}$ as in (Baheti, et al., 2012) for the arrangement of Archimedean spiral multi-turn four arms. It is a consideration that in certain cases the spaced spiral spacing increases or spiral turns can decrease the minimum frequency of the design. And the S11 parameter decreases by decreasing the width of the spiral cable. (Du et al., 2009) Meanwhile, making excessive amounts of turn can increase the resistive radio frequency (RF) ohmic losses in (Alhayali, 2012). Here is one example of the role of optimization in finding the right number of turns. Good substrate materials and matching impedance techniques can improve spiral antenna performance, resulting from standing wave reduction achieved by using a multi-stage tapered impedance transformer.

A balanced mode can be formed from a 2-arms spiral where between the two arm there is a 180degree phase difference and the same amplitude. Meanwhile, it is found in (Nakano et al., 2009) that the 2-arms unbalanced spiral indicates a wellaccepted VSWR in the design frequency range. Gains obtained are similar in spiral balanced mode, also obtained circular polarization. In this case, the unbalanced mode is a simple design type because without the need for a balun circuit. However, the unbalanced mode can actually lower the gain and efficiency of the antenna when there are nulls in the radiation pattern. This occurs in dual-polarized 2arms configurations printed on top-down printed circuit boards (PCBs), for the reflection mitigation of spiral arm currents. (Kunysz, W.) In this case, trade-offs need to be considered.

When the technical gap loading is used to lower the initial resonance frequency, when compared to the traditional Archimedean spiral antennas at the same lowest working frequency then the proposed antenna area can be reduced by more than $30 \%$, while the gain remains almost the same. (Liu et al., 2008)

Another issue with the spiral antenna lies in the reflection of energy at the end of the spiral that has not been irradiated out but instead is emitted back with opposite polarization as the current flows back to the source excitation port. Therefore, how to weaken and/or absorb this energy near the reflection area becomes an interesting investigative area if supported by appropriate optimization techniques, of course, while still working to reduce complexity and maintain costs.

\section{CONCLUSIONS}

The identification and analysis of the wideband spiral antenna have been done and given the fact that communication is increasingly requiring large bandwidths then the frequency independent becomes the main driving character of the further development of spiral antennas.

Optimization of spiral antenna system dimension design, where optimization time is getting faster with an optimization algorithm, correct spiral material selection, supported by certain numerical calculation plays an important role in improving spiral antenna performance. In this case, sustainable research and development also play a strategic role to increase property such as bandwidth expansion, HPBW, gain, axial ratio, circular polarization, radiation pattern, impedance, return loss and reflection coefficient in spiral antenna application expansion.

\section{REFERENCES}

Alaydrus, M., 2015. "Antenna research - state of the art," Jurnal Incomtech, vol.6, no.1, pp.1-22. Viewed 3 May 2017, http://publikasi.mercubuana.ac.id/index. $\mathrm{php} /$ Incomtech /article/view/1146.

Alhayali, A.N.R.J., 2012. "Design of a planar spiral antenna in the $5.5-6.8 \mathrm{ghz}$ frequency range using moment method", Tikrit Journal of Pure Science, University of Mosul, vol.17, no. 4. 
Alhorr, F.S., 2007. UWB single arm spiral antenna on an ebg substrate, thesis, Texas Tech University.

Baby, L. \& Michael, N.R., 2015. "Self-complementary two arm archimedean spiral antenna for aircraft applications", International Journal of Current Engineering and Technology, vol.5, no.5.

Baheti, A., Mehrabani, A.,M. \& Shafai, L., 2012. "Analysis of multi-turn 4-arm archimedean spiral antenna with varying spacing between arms", PIERS proceedings, Kuala Lumpur.

Balanis, C.A., 2005. Antenna Theory, Wiley. New Jersey, 3rd ed.

Dongre, V.J. \& Takle, M.C., 2014. "Multiple band monopole arm microstrip spiral antenna for cognitive radio", Global Journal of Research In Engineering: F Electrical and Electronics Engineering, vol. 14, no.3.

Du, Y.X., Qin, L., and Xi, X.L., 2009. "The Analysis and Simulation of microstrip Spiral Antenna for Microwave Hyperthermia", viewed 30 November 2017, http://www.electro-tech-online.com/attachments /the-analysis-and-pdf.40323/

Hidayat, R. , 2016. "Circular patch design and analysis for rfid tag antenna application with back propagation algorithm of artificial neural network", Proceeding of SNAPP : sains and technology, Bandung Islamic University, vol.6, no.1. Viewed 15 August 2017, http:// proceeding.unisba.ac.id/index.php /sains teknologi /article/view/655.

Hinostroza, I., Guinvarc'h, R. \& Haupt, L.R., 2011. "The design of wide band planar arrays of spiral antennas", IEEE AP-S Conference, Spokane, WA.

Kim1,J.-W., Min1,K.-S., Kim1,I.-H. \& Park1,C.-J., 2012. "Triple band spiral antenna for non-linear junction detector", IEEE Proceedings of ISAP2012, Nagoya, Japan.

Kim, H. B., Hwang, K. C. \& Kim, H. S., 2013. "Cavitybacked two arm spiral antenna with a ring-shaped absorber for partial discharge diagnosis", $J$ Electr Eng Technol, vol.8, no.4, pp.856-862. http://dx.doi.org/10.5370/JEET.2013.8.4.856.

Klimya, T.S. \& Prakash, A.K., 2015. "Cavity backing in spiral antennas", International Journal of Engineering Research and General Science, Toc H Institute of Science and Technology, vol.3, no.1.

Kunysz, W. Novel Method of Mitigation Reflections from Antenna Spiral Arm Ends. Quantum Reversal Inc., White paper, viewed 30 November 2017, $\mathrm{http} / /$ quantumreversal.com/docs/ method-mitigationreflections-antenna.pdf.

Liu, C., Lu, Y., Du, C., Cui, J. \& Shen, X., 2010. "The broadband spiral antenna design based on hybrid backed-cavity", IEEE Transactions on Antennas and Propagation, vol. 58, no. 6.

Liu, Q., Ruan, C.-L. , Peng, L. \& Wu, W.-X., 2008. “A novel compact archimedean spiral antenna with gaploading", Progress In Electromagnetics Research
Letters, University of Electronic Science and Technology of China ,vol.3, pp.169-177.

Mohamad, S., Cahill, R. \& Fusco, V., 2015. "Performance of archimedean spiral antenna backed by fss reflector", Electronics Letters, Queen's University Belfast, vol.51, no.1, pp.14-16. DOI: 10.1049/el.2014.3693.

Morais, J.H.C. \& Silva, S.G., 2013. "Square-spiral antenna with unbalanced-excitation", XXXI Brazilian Symposium on Telecommunications. Viewed 30 November 2017, https://www.researchgate.net/ publication/269226225_Square-spiral_Antenna_with_ Unbalanced-Excitation.

Muñoz, L.E.G., Lavado, A.R., Aller, M.M., Puente, J.M.S., Fernández, J.A.L. \& Vargas, D.S., 2013. "Ultra-wideband conical spiral antenna for vlbi2010, XXXIII Finnish URSI Convention on Radio Science and SMARAD Seminar, Universidad Carlos III de Madrid.

Nakano, H., Igarashi, T., Oyanagi, H., Iitsuka, Y. \& Yamauchi, J., 2009. "Unbalanced-mode spiral antenna backed by an extremely shallow cavity", IEEE Transactions On Antennas and Propagation, vol. 57, no. 6 .

Patil, S., Vyas, C. \& Khalore, A., 2016. "Spiral antennas for communication engineering applications: a systematic approach. International Journal of Innovative Research in Electronics and Communications (IJIREC), vol.3, no.2.

Rahman, N., Sharma, A., Afsar, M., Palreddy, S. \& Cheung, R., 2011. "Dielectric characterization and optimization of wide-band, cavity-backed spiral antennas", ACES Journal, vol.26, no. 2

Rupčić, S., Radivojević, V.M. \& Grgić, K., 2016. "Loxodromic antenna array based on archimedean spiral", International Journal of Electrical and Computer Engineering Systems, vol.7, no.1.

Schreider, L. , Begaud, X. , Soiron, M. , Perpere, B. \& Renard, C., 2007. "Broadband archimedean spiral antenna above a loaded electromagnetic band gap substrate", IET Microwave, Antennas Propagation, vol.1, no.1, pp.212-216. doi:10.1049/ietmap:20050323.

Shamii, Y.R., Kovitz, J.M. \& Rajagopalan, H., 2012. "Nature-inspired optimization techniques in communication antenna design", Proceeding of the IEEE, vol.100, no.7.

Stutzman, W.L. \& Thiele, G.A., 1998. Antenna Theory and Design, John Wiley\& Sons,Inc. New York, $2^{\text {nd }}$ ed. 